\title{
Isolated Choroid Plexus Granulomas: Initial Presentation of Neurosarcoidosis?
}

\author{
Michael E. Kallen, Kritsanapol Boon-Unge, William H. Yong, Whitney B. Pope, John G. Frazee, \\ Harry V. Vinters
}

Can J Neurol Sci. 2014; 41: 112-114

Granulomatous mass lesions in the central nervous system (CNS) are most commonly associated with neurosarcoidosis and tuberculosis ${ }^{1}$. The differential diagnosis also includes primary angiitis of the CNS, Wegener's granulomatosis, idiopathic chronic hypertrophic pachymeningitis, xanthogranulomas, Langerhans cell histiocytosis, and infections resulting from various viral and parasitic agents. These entities do not commonly occur as an isolated single mass within the choroid plexus. Neurosarcoidosis has been rarely reported as an isolated lesion within this site $^{2}$. Additionally, an uncommon entity, pathogen-free granulomatous disease (PFGD) of the CNS describes granulomatous disease confined to the $\mathrm{CNS}^{1}$ but without systemic evidence of sarcoidosis.

We present an unusual case of an isolated granulomatous mass of the right lateral ventricle involving the choroid plexus, in an otherwise healthy patient with minimal significant past medical history.

A 63-year-old woman was referred with insidious onset of malaise, headaches, and vision changes. She subsequently developed focal neurological symptoms including twitching of the left hand. Brain magnetic resonance imaging (MRI) (see Figure 1) showed an enhancing lesion involving the right choroid plexus centered in the atrium of the right lateral ventricle, measuring $28 \times 15 \mathrm{~mm}$. The MRI noted periventricular edema of the white matter with mild dilatation of the right lateral ventricle. The patient underwent uncomplicated resection of the lesion.

Computed tomogram (CT) scan of the chest showed approximately ten pleural and fissure-based micronodules, some with "ground glass" appearance, with no significant hilar lymphadenopathy. The suggestion of a prominent subcarinal and pretracheal node was noted. Transbronchial biopsy of the right upper and lower lung lobes was performed, showing fragments of normal lung tissue with no significant inflammation or granulomas, and no evidence of infection or malignancy.

Laboratory findings were notable for negative anti-neutrophil cytoplasmic antibodies (ANCA), positive Mycobacterium tuberculosis (MTB)-quantiferon, Erythrocyte Sedimentation Rate (ESR) $20 \mathrm{~mm} / \mathrm{hr}$ (reference range 0-22 mm/hr), C-Reactive Protein $(\mathrm{CRP})<0.5 \mathrm{mg} / \mathrm{dL}$ (reference range $<0.8 \mathrm{mg} / \mathrm{dL}$ ), Angiotensin-Converting Enzyme (ACE) $44 \mathrm{U} / \mathrm{L}$ (reference range 10-66 U/L).

The patient had been born in Mexico and moved to the USA in the 1960s. She had smoked tobacco in the past but discontinued smoking in 2011, and other than a distant history of factory work, has no obvious environmental or occupational exposures.
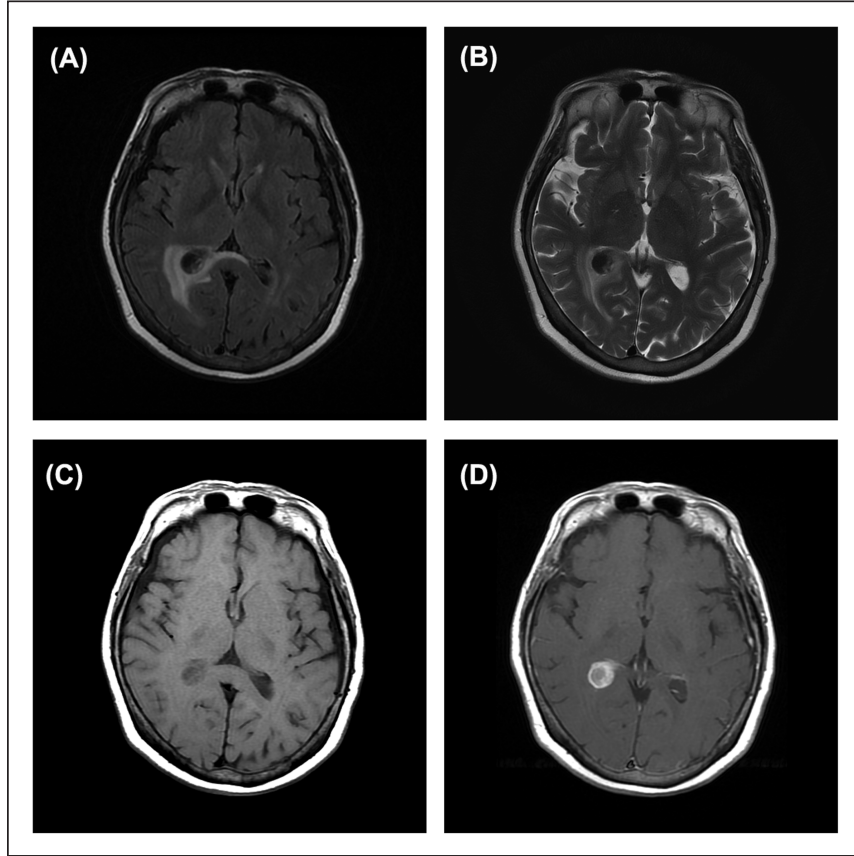

Figure 1: MRI axial images of the brain showing a T1 and T2 hypointense mass with avid contrast enhancement located within the atrium of the right lateral ventricle and involving the choroid plexus. There is vasogenic edema of the peri-atrial white matter best seen on T2-weighted images. (A) FLAIR (B) T2 (C) T1 without contrast (D) T1 with contrast.

One year after the operation, the patient had resolution of her malaise, headaches, and focal neurologic symptoms. Postoperative CT scans showed mild gliosis at the resection margin, but no regrowth of the mass or new intracranial lesions. She has no new signs or symptoms of sarcoidosis, or of any neurologic disease, but is being treated for presumed neurosarcoidosis with

From the Department of Pathology and Laboratory Medicine (MEK, KBU, WHY, HVV), Jonsson Comprehensive Cancer Center (WHY), Department of Radiologica Sciences (WBP), Department of Neurosurgery (JGF), Department of Neurology (HVV), David Geffen School of Medicine at the University of California Los Angeles, Los Angeles, Los Angeles, California, USA.

Received June 10, 2013. Final Revisions Submitted July 22, 2013 Correspondence to: Michael E. Kallen, UCLA Pathology \& Lab Medicine, Box 951732, A7-215 CHS, Los Angeles, California, 90095-1732, USA.

Email: mkallen@mednet.ucla.edu. 


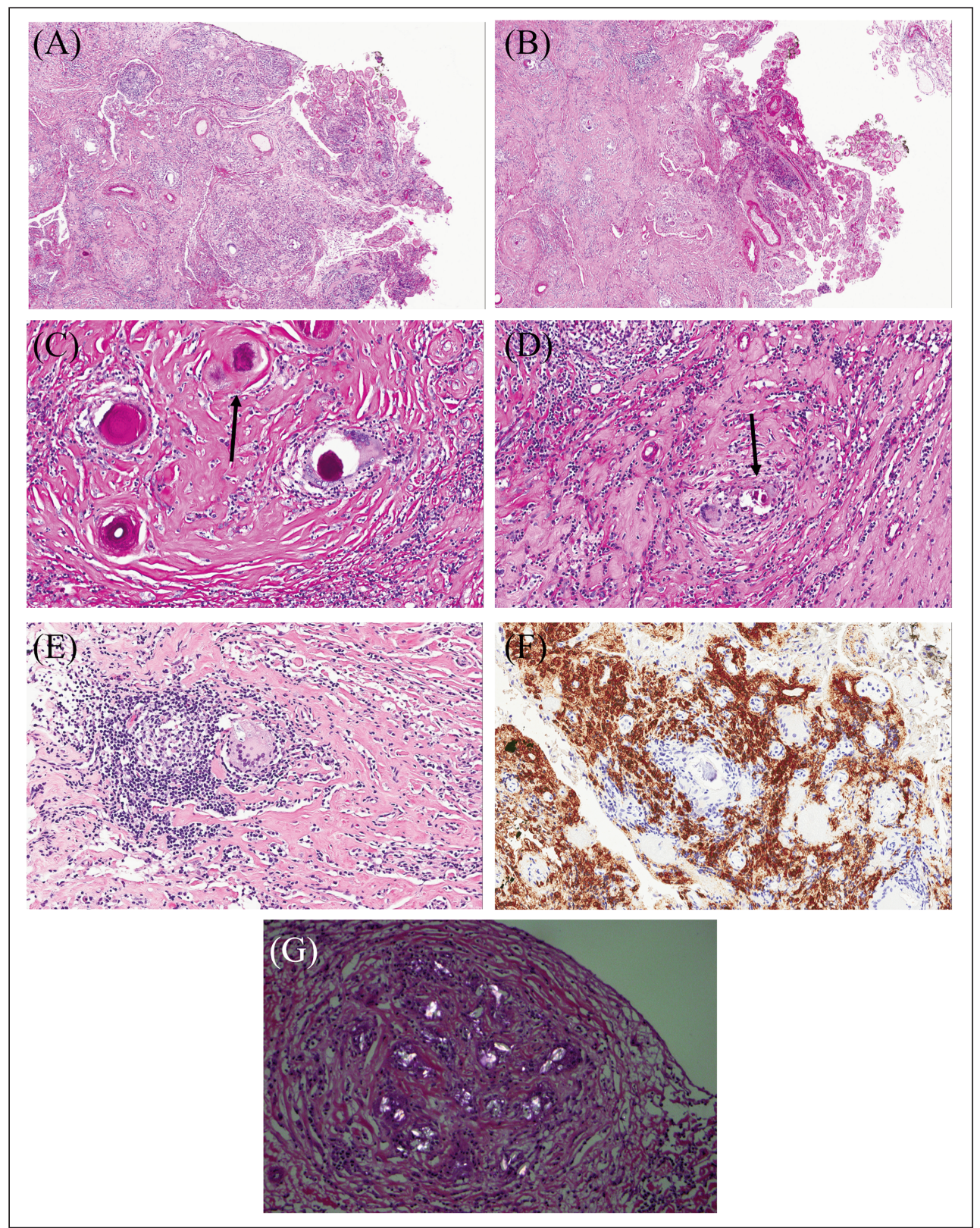

Figure 2: Representative histologic sections showing chronically inflamed connective tissue with numerous granulomas and prominent Schaumann bodies. (A) and (B) show low power views (4x) of the granulomatous inflammation and residual choroid plexus. $(C)$ and $(D)$ show Schaumann bodies, marked with arrows (20x). (E) shows a magnified (20x) view of a granuloma. (F) CD138 highlights plasma cells (20x). (G) Section viewed under polarized light shows refractile material within giant cells (40x).

low dose hydroxychloroquine, and for possible latent tuberculosis infection with Isoniazid.

The specimen consisted of a $3.5 \times 1.3 \times 1.2 \mathrm{~cm}$ wellcircumscribed firm soft tissue fragment with a homogeneous cut surface. Histologic sections showed dense, fibrous, chronically inflamed connective tissue with numerous granulomas. Many of the granulomas contained prominent Schaumann bodies (see Figure 2C and D), while others showed giant cells with large cytoplasmic vacuoles. Some giant cells showed calcified and focally refractile cytoplasmic material (see Figure $2 \mathrm{G}$ ). There was no evidence of necrosis. Fragments of residual benign choroid plexus epithelium were identified within the mass. Remote from the granulomas, dense spherical calcium aggregates were identified, probably representing psamomma bodies.

Immunohistochemical stains were supportive of granulomatous inflammation. The CD68 stain highlighted histiocytes forming granulomas. The CD20 stain highlighted diffusely scattered and perivascular B-lymphocytes. The CD138 stain highlighted scattered plasma cells (see Figure $2 \mathrm{~F}$ ). The 
IgG4 stain highlighted rare scattered positive cells. The CD3 stain highlighted T-lymphocytes surrounding granulomas and blood vessels. The Congo Red stain showed no significant amyloid deposition. Gomori Methenamine Silver (GMS) and Periodic Acid-Schiff (PAS) stains were negative for fungal organisms, as was the acid fast stain. Additional polymerase chain reaction for Mycobacterium tuberculosis complex was performed on sections from the paraffin block, at the University of Washington, Department of Laboratory Medicine and Pathology; no Mycobacterium tuberculosis complex DNA was detected.

This specimen shows histologic evidence of non-caseating granulomas with prominent Schaumann bodies, suggestive of neurosarcoidosis in a most unusual location. The clinical features of the case, including the patient's age, make neurosarcoidosis an unlikely diagnosis. Sarcoidosis affects the CNS in 5-26\% of patients $^{3,4}$. Although most patients with neurosarcoidosis have systemic disease, isolated CNS involvement can be seen in an estimated less than $1 \%$ of cases $^{4,5}$. Our patient had no evidence of systemic involvement by sarcoidosis. We acknowledge the possibility that the "negative" lung biopsy may be a false negative secondary to sampling error.

While sarcoidosis can affect almost any part of the neuraxis, it is primarily a leptomeningeal disease with a predilection for the basal cisterns. Other common areas of involvement are the leptomeninges at the base of the brain and posterior fossa, cranial nerves, and adjacent brain parenchyma via local extension ${ }^{3}$. Pathogen-free granulomatous disease of the CNS is a rare entity used to describe CNS granulomatous lesions in individuals who do not have other features of sarcoidosis ${ }^{1}$. The diagnosis of PGFD is made by the exclusion of other clinical entities, including neurosarcoidosis and primary angiitis of the CNS. It can be difficult to distinguish between neurosarcoidosis and PFGD, because there are no reliable diagnostic tests, and because isolated granulomas may be the first manifestation of systemic sarcoidosis ${ }^{1}$. Therefore, clinical presentations and patterns of CNS involvement are used to classify unclear cases, although a subset of cases remains problematic. Thomas et al found PFGD to have a worse prognosis than neurosarcoidosis, and to have the characteristic defining feature of widespread meningeal and parenchymal granulomatous inflammation, sometimes with spinal cord involvement ${ }^{1}$. Additionally, PFGD commonly presents with seizures, probably reflective of diffuse cortical inflammation in contrast to the focality of sarcoid granulomas. Our patient's clinical presentation therefore does not fit with described cases of PFGD, but may represent an anatomically localized example of this entity.

Although neurocysticercosis is a possible cause of granulomatous inflammation in this region of the United States, the intraventricular location makes this less likely, and there were no other histologic or clinical signs of such an infection. This case shows histologic evidence of non-caseating granulomas with notable Schaumann bodies, suggestive of neurosarcoidosis apparently confined to the choroid plexus. In the absence of other stigmata of sarcoid, this could be a rare presentation of isolated neurosarcoidosis or PFGD of the CNS.

\section{ACKNOWLEDGEMENTS}

Each listed author has contributed to this manuscript as follows:

MK-clinical data, obtaining and editing histologic images, writing manuscript. KBU-obtaining and editing histologic images, editing manuscript. WY-editing manuscript. WPproviding radiology images, editing manuscript. JF-obtaining neurosurgical specimen, editing manuscript. HV-providing case information, editing manuscript.

\section{REFERENCES}

1. Thomas G, Murphy S, Staunton H, O'Neill S, Farrell MA, Brett FM. Pathogen-free granulomatous diseases of the central nervous system. Hum Pathol. 1998;29:110-5.

2. Strefling AM, Summerville JW, Urich H. Involvement of choroid plexuses in neurosarcoidosis. Acta Neuropathol. 1987;74:402-4.

3. James DG, Sharma OP. Neurosarcoidosis. Proc R Soc Med. 1967; 60:1169-70.

4. Smith JK, Matheus MG, Castillo M. Imaging manifestations of neurosarcoidosis. Am J Roentgenol. 2004;182:289-95.

5. Brinar VV, Habek M. Isolated central nervous system sarcoidosis: a great mimicker. Clin Neurol Neurosurg. 2008;110:939-42. 